\title{
Two-Level Incentive Stackelberg Strategies in LQ Differential Games with Two Noncooperative Leaders and One Follower
}

\author{
Koichi MizukAm* and Hansheng Wu*
}

This paper mainly is concerned with the derivation of the sufficient conditions for the two-level incentive stackelberg strategies in the two-level hierarchical differential games with two noncooperative leaders and one follower, characterized by a kind of linear state dynamics and quadratic cost functionals. In the present paper, we first define a concept of "team-optimal Nash equilibrium solution", and by a simple numerical example, show a general method solving such a static two-level incentive game in which two leaders find their "team-optimal Nash equilibrium solution" by announcing their strategies. Then, we construct the two-level incentive Stackelberg strategies $\bar{\gamma}_{1} \in \Gamma_{1}$ and $\bar{\gamma}_{2} \in \Gamma_{2}$ of the form

$$
\bar{\gamma}_{1}\left(x(t), u_{31}(t), t\right)=\eta_{10}(t) x(t)+\eta_{11}(t) u_{31}(t),
$$$$
\bar{\gamma}_{2}\left(x(t), u_{32}(t), t\right)=\eta_{20}(t) x(t)+\eta_{22}(t) u_{32}(t),
$$

for the players $\mathbf{P} \mathbf{1}$ and $\mathbf{P} 2$ (leaders) to achieve their "team-optimal Nash equilibrium solution" in such a game characterized by a kind of linear state dynamics and quadratic cost functionals, and also obtain the sufficient conditions which are satisfied by these twolevel incentive Stackelberg strategies $\bar{\gamma}_{1} \in \Gamma_{1}$ and $\bar{\gamma}_{2} \in \Gamma_{2}$.

Key Words: Stackelberg games, incentive Stackelberg strategy, team/game theory, Nash equilibrium solution, linear quadratic system

\section{Introduction}

In two-player differential games, existence of a hierarchy in decision-making implies that one of the players is in a position to determine his strategy ahead of time, announce it and enforce it on the other player; therefore, the Stackelberg

\footnotetext{
* Faculty of Integrated Arts and Sciences, Hiroshima University, 1-1-89, Higashisenda-machi, Naka-ku, Hiroshima, Japan

(Received October 24, 1986)

(Revised January 20, 1987)
}

solution is the only possible hierarchical equilibrium solution applicable in such decision-making problems, called Stackelberg games. A hierarchical equilibrium solution in Stackelberg games is generally called a Stackelberg strategy. However, an incentive Stackelberg strategy is the announced (closed-loop) strategy by which a leader can achieve his team-optimal solution in a Stackelberg game. In the recent years, there were many papers and works dealing with the incentive Stackelberg strategy. In Ref. 1), many aspects of the incentive problems were investigated. In Ref. 2), an approach to obtaining the closedloop Stackelberg solution of linear quadratic dynamic games was developed. In Ref. 3), another method to construct the Stackelberg strategy was presented with rather relaxed restriction. In Ref. 4), by using a geometric approach, the existence and derivation of affine incentive Stackelberg strategy were addressed. In Ref. 5), Zheng, Basar and Cruz gave an extensive discussion and derivation of closed-loop Stackelberg strategies and incentive policies in dynamic decision-making problems of some types. In Ref. 6), the sufficient conditions for the incentive Stackelberg strategies in linear quadratic differential games were derived. In Refs. 7) 9), the concept of "inducible region" was introduced and some applications of this concept to the multistage Stackelberg games were given.

The incentive games (i. e., Stackelberg games in which the leader seek his team-optimal solution) with two players can be extended to the ones with three players in two-level or three-level hierarchy (see Ref. 1)). For the case with three-level 


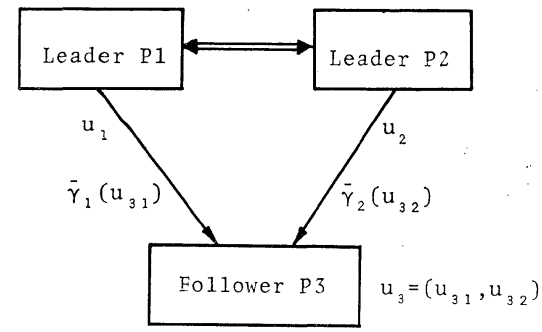

Fig. 1 Hierarchical structure with two noncooperative leaders and one follower

hierarchy, there were several works. In Ref. 10), for example, the sufficient condition for such a three-level incentive Stackelberg strategy in a linear quadratic discrete 'game was obtained. In Ref. 11), Ishida and Shimemura defined a threelevel incentive Stackelberg strategy in a non-linear differential game, and derived the sufficient conditions for such a three-level incentive Stackelberg strategy in a linear quadratic differential game. In Ref. 9), the three-level incentive Stackelberg decision-making problems were studied by using the inducible region concept presented in Ref. 8).

In the present paper, we consider in detail another form of Stackelberg games, which has not been considered by the authors mentioned here in Refs. 1) 11), so far. That is, we will only consider the incentive Stackelberg games with three players in two-level hierarchy. Furthermore, we will only discuss the two-level hierarchical games with two noncooperative leaders and one follower. That is, we consider the hierarchical structure depicted in Fig. 1, where there are three players $\mathbf{P} 1, \mathbf{P} \mathbf{2}$ and $\mathbf{P} \mathbf{3} ; \mathbf{P} \mathbf{1}$ and $\mathbf{P} \mathbf{2}$ are two leaders, and $\mathbf{P} 3$ follower, under the stipulation that the two leaders act noncooperatively. The hierarchical structure depicted by Fig. 1 means that $\mathbf{P} \mathbf{1}$ and P 2 announce their strategies respectively, ahead of time to another player, then the player $\mathbf{P} 3$, in view of the strategies of $\mathbf{P} \mathbf{1}$ and $\mathbf{P} \mathbf{2}$, decides his optimal decision. Here, two leaders $\mathbf{P} \mathbf{1}$ and $\mathbf{P} \mathbf{2}$ are under the noncooperative mode of action. Specifically, we stipulate that two leaders choose their decisions under Nash equilibrium solution concept. In the subsequent parts of this paper, we will call the game ordered in such a way a two-level hierarchical game with two noncooperative leaders and one follower.

In this paper, we discuss the two-level hierarchical game with two noncooperative leaders and one follower, characterized by a kind of linear state dynamics and quadratic cost functionals. Being different from the methods used in other papers, we construct new form of the two-level incentive Stackelberg strategies $\bar{\gamma}_{1}$ and $\bar{\gamma}_{2}$ for the players $\mathbf{P} \mathbf{1}$ and $\mathbf{P} \mathbf{2}$ to achieve their "team-optimal Nash equilibrium solution" (in the sense of what is shown in Section 2) in this game, and also obtain the sufficient conditions which are satisfied by these two-level incentive Stackelberg strategies $\bar{\gamma}_{1}$ and $\bar{\gamma}_{2}$.

In the next section, we give some concepts in the two-level hierarchical game with two noncooperative leaders and one follower, and show a general method solving such a two-level incentive game problem by a simple numerical example. In Section 3, we define the two-level incentive Stackelberg strategies $\bar{\gamma}_{1}$ and $\bar{\gamma}_{2}$ of two leaders $\mathbf{P} \mathbf{1}$ and $\mathbf{P} \mathbf{2}$ in such a differential game characterized by a kind of linear state dynamics and quadratic cost functionals, and state the main steps by which we can derive the sufficient conditions for the strategies $\bar{\gamma}_{1} \in \Gamma_{1}$ and $\bar{\gamma}_{2} \in \Gamma_{2}$ in this differential game. Section 4 gives this sufficient condition, and this condition is derived in detail. The paper will be concluded in Section 5 with a very brief discussion on the above results and some possible further research.

\section{Two-Level Hierarchical Games with Two Noncooperative Leaders and One Follower}

In this section, we would give some concepts in the two-level hierarchical games with two noncooperative leaders and one follower, and show a general method solving such a game problem by a simple numerical example.

\subsection{Team-optimal Nash equilibrium solution}

For the two-level hierarchical game with two noncooperative leaders and one follower, we will assume that there are three players P 1, P 2 and $\mathbf{P} 3$; $\mathbf{P} 1$ and $\mathbf{P} 2$ are two noncooperative leaders, P 3 follower. Furthermore, we assume that the 
cost functions of $\mathbf{P} \boldsymbol{i}, \quad i=1,2,3$, are respectively $L_{i}\left(u_{1}, u_{2}, u_{31}, u_{32}\right)$, where $u_{1} \in \boldsymbol{R}^{m_{1}}$ and $u_{2} \in \boldsymbol{R}^{m_{2}}$ are respectively the decisions of $\mathbf{P} \mathbf{1}$ and $\mathbf{P} 2$, and $u_{3}=\left(u_{31}, u_{32}\right) \in \boldsymbol{R}^{m_{3}}$ (here, $\left.m_{3}=m_{31}+m_{32}\right)$ is $\mathbf{P} 3$ 's decision. Thus we have the following definition.

Definition 1. A two-tuple of decision $\left\{\left(u_{1}^{*}, u_{31}^{*}\right)\right.$, $\left.\left(u_{2}^{*}, u_{32}^{*}\right)\right\}$ with $\left(u_{i}^{*}, u_{3 i}^{*}\right) \in \boldsymbol{R}^{m_{i}+m_{3 i}}, i=1,2$, is said to constitute a "team-optimal Nash equilibrium solution" for a two-level hierarchical game with two noncooperative leaders and one follower, if the following two inequalities are satisfied for all $\left(u_{i}, u_{3 i}\right) \in \boldsymbol{R}^{m_{i}+m_{3 i} i}, i=1,2$,

$$
\begin{aligned}
& L_{1}^{*}=L_{1}\left(u_{1}^{*}, u_{2}^{*}, u_{31}^{*}, u_{32}^{*}\right) \leqq L_{1}\left(u_{1}, u_{2}^{*}, u_{31}, u_{32}^{*}\right), \\
& L_{2}^{*}=L_{2}\left(u_{1}^{*}, u_{2}^{*}, u_{31}^{*}, u_{32}^{*}\right) \leqq L_{2}\left(u_{1}^{*}, u_{2}, u_{31}^{*}, u_{32}\right) .
\end{aligned}
$$

The two-tuple of quantities $\left\{L_{1}^{*}, L_{2}^{*}\right\}$ is known as a "team-optimal Nash equilibrium outcome" of this game.

\subsection{The basic approach to solving such a two-level incentive game}

In the two-level hierarchical games with two noncooperative leaders and one follower, we also assume that

i) the players $\mathbf{P} \mathbf{1}$ and $\mathbf{P} \mathbf{2}$ announce their strategies $\gamma_{1}: \boldsymbol{R}^{m_{31} \rightarrow \boldsymbol{R}^{m_{1}}}$, and $\gamma_{2}: \boldsymbol{R}^{m_{32} \rightarrow \boldsymbol{R}^{m_{2}}}$, respectively, ahead of time to the other player $\mathbf{P} \mathbf{3}$;

ii) then $\mathbf{P} 3$ decides his optimal decision after knowing the announced strategies of $\mathbf{P} \mathbf{1}$ and $\mathbf{P} 2$.

Here, if $\mathbf{P} \mathbf{1}$ and $\mathbf{P} \mathbf{2}$ can obtain the "team-optimal Nash equilibrium solution" by their strategies $\gamma_{1}$ and $\gamma_{2}$ announced, then we call $\gamma_{1}$ and $\gamma_{2}$ two-level incentive Stackelberg strategies of $\mathbf{P} \mathbf{1}$ and $\mathbf{P} 2$, respectively, in this game. Furthermore, this two-level hierarchical game to find the twolevel incentive Stackelberg strategies $\gamma_{1}$ and $\gamma_{2}$ is called the two-level incentive game with two noncooperative leaders and one follower in this paper.

If we assume that $\left\{\left(u_{1}^{*}, u_{31}^{*}\right), \quad\left(u_{2}^{*}, u_{32}^{*}\right)\right\} \in \boldsymbol{R}^{m_{1}+m_{31}}$ $\times \boldsymbol{R}^{m_{2}+m_{32}}$, is the "team-optimal Nash equilibrium solution" of the players $\mathbf{P} \mathbf{1}$ and $\mathbf{P} \mathbf{2}$, then this two-level incentive game can be simply stated as :

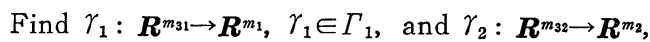
$\gamma_{2} \in \Gamma_{2}$, such that

$$
\arg \underset{u_{31}, u_{32}}{\min } L_{3}\left(\gamma_{1}\left(u_{31}\right), \gamma_{2}\left(u_{32}\right), u_{31}, u_{32}\right)
$$

$$
\begin{aligned}
& =\left(u_{31}^{*}, u_{32}^{*}\right) \\
& \gamma_{1}\left(u_{31}^{*}\right)=u_{1}^{*} \\
& \gamma_{2}\left(u_{32}^{*}\right)=u_{2}^{*}
\end{aligned}
$$

where $\boldsymbol{\Gamma}_{i}, \quad i=1,2$, is the class of admissible incentive Stackelberg strategies of $\mathbf{P} \boldsymbol{i}, i=1,2$.

Actually, the equation (2) is a sufficient condition of that $\gamma_{1}$ and $\gamma_{2}$ are the incentive Stackelberg strategies in this game.

\subsection{Example}

Finally, we will conclude this section with a illustrative example of a static game.

Consider the two-level incentive static game with two noncooperative leaders and one follower described by the following form

$$
\begin{aligned}
& L_{1}=\left(u_{1}-1\right)^{2}+2 u_{1} u_{2}+\left(u_{31}-2\right)^{2}+3 u_{32}, \\
& L_{2}=\left(u_{2}+1\right)^{2}+4 u_{1} u_{2}+u_{31}+\left(u_{32}+2\right)^{2}, \\
& L_{3}=u_{1}+u_{2}+\left(u_{31}-1\right)^{2}+\left(u_{32}-2\right)^{2}
\end{aligned}
$$

The main steps to find the two-level incentive Stackelberg strategies $\gamma_{1}\left(u_{31}\right)$ and $\gamma_{2}\left(u_{32}\right)$ of the players $\mathbf{P} \mathbf{1}$ and $\mathbf{P} \mathbf{2}$ are as follows.

i) Finding the "team-optimal Nash equilibrium solution" of $\mathbf{P} \mathbf{1}$ and $\mathbf{P} 2$, we obtain

$$
\left\{\left(u_{1}^{*}, u_{31}^{*}\right),\left(u_{2}^{*}, u_{32}^{*}\right)\right\}=\{(-2,2),(3,-2)\} .
$$

ii) Here, let us consider the two-level incentive Stackelberg strategies $\gamma_{1}\left(u_{31}\right)$ and $\gamma_{2}\left(u_{32}\right)$ of the form

$$
\begin{aligned}
& u_{1}=\gamma_{1}\left(u_{31}\right)=u_{1}^{*}+k_{1} \cdot\left(u_{31}-u_{31}^{*}\right), \\
& u_{2}=\gamma_{2}\left(u_{32}\right)=u_{2}^{*}+k_{2} \cdot\left(u_{32}-u_{32}^{*}\right),
\end{aligned}
$$

where $k_{1}$ and $k_{2}$ are two undetermined coefficients.

Substituting for $u_{1}^{*}, u_{2}^{*}, u_{31}^{*}$ and $u_{32}^{*}$ from (4) in (5), further, we have

$$
\begin{aligned}
& u_{1}=\gamma_{1}\left(u_{31}\right)=-2+k_{1} \cdot\left(u_{31}-2\right) \\
& u_{2}=\gamma_{2}\left(u_{32}\right)=3+k_{2} \cdot\left(u_{32}+2\right) .
\end{aligned}
$$

iii) Solve the following problem:

$$
\begin{array}{ll}
\min _{u_{31}, u_{32}} & L_{3}\left(u_{1}, u_{2}, u_{31}, u_{32}\right) \\
\text { s. t. } & u_{1}=\gamma_{1}\left(u_{31}\right)=-2+k_{1} \cdot\left(u_{31}-2\right), \\
& u_{2}=\gamma_{2}\left(u_{32}\right)=3+k_{2} \cdot\left(u_{32}+2\right) .
\end{array}
$$

Since $L_{3}$ is strictly convex in $u_{31}$ and $u_{32}$, the necessary and sufficient conditions for the problem above are given as follows.

$$
\begin{aligned}
& \left(\partial L_{3} / \partial u_{31}\right)=k_{1}+2\left(u_{31}-1\right)=0, \\
& \left(\partial L_{3} / \partial u_{32}\right)=k_{2}+2\left(u_{32}-2\right)=0 .
\end{aligned}
$$

iv) When we choose that $k_{1}=-2$ and $k_{2}=8$, then both $u_{31}^{*}=2$ and $u_{32}^{*}=-2$ must be the solution of the problem given in iii). Thus, we can 
obtain the two-level incentive Stackelberg strategies of $\mathbf{P} \mathbf{1}$ and $\mathbf{P} \mathbf{2}$ as follows.

$$
\begin{aligned}
& \gamma_{1}\left(u_{31}\right)=-2 u_{31}+2, \\
& \gamma_{2}\left(u_{32}\right)=8 u_{32}+19 .
\end{aligned}
$$

Factly, by the strategies ( 9 ) announced ahead of time, the cost functional of $\mathbf{P} \mathbf{3}$ will become

$$
L_{3}\left(\gamma_{1}\left(u_{31}\right), \gamma_{2}\left(u_{32}\right), u_{31}, u_{32}\right)=\left(u_{31}-2\right)^{2}+\left(u_{32}+2\right)^{2}+18
$$

By (10), the player P3's optimal decision must be that $u_{31}^{*}=2$ and $u_{32}^{*}=-2$. Thus, the strategies $\gamma_{1}\left(u_{31}\right)$ and $\gamma_{2}\left(u_{32}\right)$ given by $(9)$ satisfy the condition (2), they are then indeed the two-level incentive Stackelberg strategies announced by $\mathbf{P} 1$ and $\mathbf{P} \mathbf{2}$ in this game.

In the subsequent parts of this paper, using the idea stated in this section, we will mainly discuss the two-level incentive differential games with two noncooperative leaders and one follower, characterized by a kind of linear state dynamics and quadratic cost functionals.

\section{Two-level Incentive Stackelberg Strat- egies in a Kind of LQ Differential Games}

In this section, we consider a two-level hierarchical game with two noncooperative leaders and one follower, characterized by a kind of linear state dynamics and quadratic cost functionals. The evolution of this game is described by the following linear differential equations:

$$
\begin{aligned}
& \dot{x}(t)=A(t) x(t)+\sum_{i=1}^{2}\left\{B_{i}(t) u_{i}(t)+B_{3 i}(t) u_{3 i}(t)\right\}, \\
& x\left(t_{0}\right)=x_{0}, \quad t \in\left[t_{0}, t_{1}\right]
\end{aligned}
$$

where $x(t) \in \boldsymbol{R}^{n}$ is the state vector, $u_{i}(t) \in \boldsymbol{R}^{m_{i}}, i=$ 1,2 , are the player $\mathbf{P} \boldsymbol{i}$ 's decision variable, and $u_{3}(t)=\left(u_{31}(t), u_{32}(t)\right) \in \boldsymbol{R}^{m_{31}+m_{32}}$ is $\mathbf{P} 3$ 's decision variable. $A(t), B_{i}(t), i=1,2$, and $B_{3}(t)=\left(B_{31}(t), B_{32}(t)\right)$ are matrices of appropriate dimensions and with components piecewise continuous on the time interval $\left[t_{0}, t_{1}\right]$.

Here, we assume that

i) the players $\mathbf{P} \mathbf{1}$ and $\mathbf{P} 2$ announce respectively their strategies of the following form ahead of time to the other player P3,

$$
\begin{aligned}
u_{1}(t) & =\gamma_{1}\left(x(t), u_{31}(t), t\right) \\
& =\eta_{10}(t) x(t)+\eta_{11}(t) u_{31}(t),
\end{aligned}
$$

$$
\begin{aligned}
u_{2}(t) & =\gamma_{2}\left(x(t), u_{32}(t), t\right) \\
& =\eta_{20}(t) x(t)+\eta_{22}(t) u_{32}(t),
\end{aligned}
$$

where $\eta_{i 0}(t) \in \boldsymbol{R}^{m_{i} \times n}, \quad i=1,2$, and $\eta_{i i}(t) \in \boldsymbol{R}^{m_{i} \times m_{3 i}}$, $i=1,2$, are strategy parameter matrices and their components are piecewise continuous functions of time on the interval $\left[t_{0}, t_{1}\right]$;

ii) then P3 decides his optimal strategy, after knowing the announced strategies of $\mathbf{P 1}$ and P2;

iii) $\mathbf{P} \mathbf{1}$ and $\mathbf{P} \mathbf{2}$ at same level act noncooperatively, and choose their decisions under the "teamoptimal Nash equilibrium solution" concept.

Here, we call the game ordered in this way a two-level hierarchical differential game with two noncooperative leaders and one follower. In this differential game, the cost functionals of the players $\mathbf{P} i, i=1,2,3$, are given by $J_{i}, i=1,2,3$, respectively, where

$$
\begin{aligned}
J_{i}= & \frac{1}{2} \cdot x^{T}\left(t_{1}\right) F_{i}\left(t_{1}\right) x\left(t_{1}\right)+\int_{t_{0}}^{t_{1}}\left\{x^{T}(t) Q_{i}(t) x(t)\right. \\
+ & \left.\sum_{j=1}^{2}\left[u_{j}^{T}(t) R_{i j}(t) u_{j}(t)+u_{3 j}^{T}(t) R_{3 i}(t) u_{3 j}(t)\right]\right\} d t \\
& (i=1,2,3,)
\end{aligned}
$$

where the matrices $Q_{i}=Q_{i}^{T} \geqq 0, \quad R_{i j}=R_{i j}^{T} \geqq 0$, $R_{3 i j}=R_{3 i j}^{T} \geqq 0, \quad i=1,2,3, \quad j=1,2$, and $R_{i i}>0, R_{3 i i}$ $>0, i=1,2$, are piecewise continuous functions of time on the interval $\left[t_{0}, t_{1}\right]$; and the matrices $F_{i}$ $\left(t_{1}\right)=F_{i}^{T}\left(t_{1}\right) \geqq 0$. The time interval $\left[t_{0}, t_{1}\right]$ is fixed.

We also assume that each player has access to the perfect state information and can utilize it in the choice of his decision. Further, we denote the space of admissible strategies for the players $\mathbf{P} 1$ and $\mathbf{P} 2$ by $\Gamma_{1}$ and $\Gamma_{2}$, respectively; and let P3's strategy space $\Gamma_{3}$ be $\boldsymbol{R}^{m_{31}+m_{32}}$. For each pair $\left(\gamma_{1}, \gamma_{2}, u_{3}\right) \in \Gamma_{1} \times \Gamma_{2} \times \Gamma_{3}$ (i.e., for $\eta_{i 0}, \eta_{i i}, \quad i=1,2$, and $u_{3}$ ), the linear differential equation (11) has a unique solution on $\left[t_{0}, t_{1}\right]$, for all $x_{0} \in \boldsymbol{R}^{n}$, and the values of $J_{i}, i=1,2,3$, are well defined.

In such a two-level hierarchical differential game, if $\eta_{i 0}, \eta_{i i}, i=1,2$, satisfy some conditions such that the players $\mathbf{P 1}$ and $\mathbf{P} \mathbf{2}$ can obtain a "team-optimal Nash equilibrium solution" (see Definition 1 in Section 2) by $\bar{\gamma}_{1} \in \Gamma_{1}$ and $\bar{\gamma}_{2} \in$ $\Gamma_{2}$, defined in (12) and (13), we call $\bar{\gamma}_{1} \in \Gamma_{1}$ and $\bar{\gamma}_{2} \in \Gamma_{2}$ the two-level incentive Stackelberg strategies of the players $\mathbf{P} 1$ and $\mathbf{P 2}$, respectively. Further, we call this two-level hierarchical dif- 
ferential game to find the two-level incentive Stackelberg strategies the two-level incentive differential game with two noncooperative leaders and one follower, in the following parts of this paper.

In the subsequent section, we want to determine $\eta_{i 0}, \eta_{i i}, i=1,2$, such that $\bar{\gamma}_{1}$ and $\bar{\gamma}_{2}$ would be the two-level incentive Stackelberg strategies of the players $\mathbf{P 1}$ and $\mathbf{P 2}$ in this two-level incentive differential game with two noncooperative leaders and one follower. According to the sufficient condition ( 2 ), this can be done as follows.

(I) Solve the optimal decision problems of the players P1 and P2, called Problem (A), where $u_{1}(t)$ and $u_{31}(t)$ are the decision variables of the player $\mathbf{P 1}$; and $u_{2}(t)$ and $u_{32}(t)$ the player $\mathbf{P 2}$. That is, for P1 and P2, we find the "team-optimal Nash equilibrium solution $\left\{\left(u_{1}^{*}, u_{31}^{*}\right),\left(u_{2}^{*}, u_{32}^{*}\right)\right\}$ " in such a decision problem. Let $x^{*}(t)$ be the optimal trajectory corresponding to $\left\{\left(u_{1}^{*}, u_{31}^{*}\right),\left(u_{2}^{*}, u_{32}^{*}\right)\right\}$. Then let $\bar{\gamma}_{1} \in \Gamma_{1}$ and $\bar{\gamma}_{2} \in \Gamma_{2}$ be such that, respectively,

$$
\begin{aligned}
& \bar{\gamma}_{1}\left(x^{*}(t), u_{31}^{*}(t), t\right)=u_{1}^{*}(t), \\
& \bar{\gamma}_{2}\left(x^{*}(t), u_{32}^{*}(t), t\right)=u_{2}^{*}(t),
\end{aligned}
$$

for all $t \in\left[t_{0}, t_{1}\right]$.

(II) Solve the player P3's optimal decision problem with $u_{3}(t)=\left(u_{31}(t), u_{32}(t)\right)$ as decision variable and subject to $(11), u_{1}(t)=\bar{\gamma}_{1}\left(x(t), u_{31}(t), t\right)$ and $u_{2}(t)=\bar{\gamma}_{2}\left(x(t), u_{32}(t), t\right)$, called Problem $(\mathbf{B})$, i.e., minimize $J_{3}\left(u_{1}, u_{2}, u_{3}\right)$, subject to $u_{1}(t)=\bar{\gamma}_{1} \in \Gamma_{1}$, $u_{2}(t)=\bar{\gamma}_{2} \in \Gamma_{2}$, and the differential equations (11).

(III) Find the conditions such that $x^{*}(t), u_{31}^{*}(t)$ and $u_{32}^{*}(t)$ are the solution of Problem $(\mathbf{B})$. Then if these conditions are assumed to hold, the strategies $\bar{\gamma}_{1} \in \Gamma_{1}$ and $\bar{\gamma}_{2} \in \Gamma_{2}$ are the two-level incentive Stackelberg strategies of the players P1 and $\mathbf{P 2}$, respectively.

In the next section, we will solve in detail these Problem (A) and (B), and according to the steps stated above, derive the sufficient conditions for the two-level incentive Stackelberg strategies $\bar{\gamma}_{1}$ and $\bar{\gamma}_{2}$ in this two-level incentive differential game with two noncooperative leaders and one follower.

\section{Sufficient Conditions for the Two-Level Incentive Stackelberg Strategies}

Here, we consider the linear quadratic two-level incentive differential game with two noncooperative leaders and one follower described in Section 3. According to the steps (I) (III) stated in Section 3, we will derive the sufficient conditions for the two-level incentive Stackelberg strategies $\bar{\gamma}_{1} \in \Gamma_{1}$ and $\bar{\gamma}_{2} \in \Gamma_{2}$, in this game.

(I) The "team-optimal Nash equilibrium solution" of $\mathbf{P 1}$ and $\mathbf{P 2}$.

In this step, problem (A) will be as follows. For the player Pi, $i=1,2$,

$$
\begin{array}{ll}
\min _{u_{i}, u_{3 i}} & J_{i}\left(u_{1}, u_{2}, u_{31}, u_{32}\right), \\
\text { s.t. } & \dot{x}(t)=A(t) x(t)+\sum_{i=1}^{2}\left\{B_{i}(t) u_{i}(t)+B_{3 i}(t) u_{3 i}(t)\right\} \\
& x\left(t_{0}\right)=x_{0}, \quad t \in\left[t_{0}, t_{1}\right], \\
& u_{i}(t) \in \boldsymbol{R}^{m_{i}}, \\
& u_{3 i}(t) \in \boldsymbol{R}^{m_{3 i}} .
\end{array}
$$

Assume that $\left\{\left(u_{1}^{*}, u_{31}^{*}\right),\left(u_{2}^{*}, u_{32}^{*}\right)\right\}$ is a "team-optimal Nash equilibrium solution" of Problem (A) for the players $\mathbf{P} \mathbf{1}$ and $\mathbf{P} 2$, then using the results of the Nash equilibrium solution in linear quadratic differential games (see Ref. 12), Chapter 6), we can have

$$
\begin{aligned}
u_{i}^{*}(t) & =-R_{i i}^{-1}(t) B_{i}^{T}(t) K_{i}(t) x^{*}(t), \\
u_{3 i}^{*}(t) & =-R_{3 i i}^{-1}(t) B_{3 i}^{T}(t) K_{i}(t) x^{*}(t) \\
i & =1,2,
\end{aligned}
$$

where $x^{*}(t)$ is the optimal trajectory corresponding to $\left\{\left(u_{1}^{*}, u_{31}^{*}\right),\left(u_{2}^{*}, u_{32}^{*}\right)\right\}$, and $K_{i}(t), i=1,2$, are the solutions of the coupled matrix Riccati differential equations

$$
\begin{aligned}
\dot{K}_{i}(t)= & -K_{i} A-A^{T} K_{i}-Q_{i} \\
& +K_{i} \sum_{j=1}^{2}\left\{B_{j} R_{j j}^{-1} B_{j}^{T} K_{j}+B_{3 j} R_{3 j j}^{-1} B_{3 j}^{T} K_{j}\right\},
\end{aligned}
$$

$$
\begin{aligned}
K_{i}\left(t_{1}\right) & =F_{i}\left(t_{1}\right), \quad t \in\left[t_{0}, t_{1}\right], \\
i & =1,2 .
\end{aligned}
$$

Let $\phi\left(t, t_{0}\right)$ be solution of

$$
\left(\partial \phi\left(t, t_{0}\right) / \partial t\right)=\Lambda(t) \phi\left(t, t_{0}\right),
$$

$\phi\left(t_{0}, t_{0}\right)=I, \quad t \in\left[t_{0}, t_{1}\right]$,

where

$$
\Lambda(t)=A-\sum_{j=1}^{2}\left\{B_{j} R_{j j}^{-1} B_{j}^{T} K_{j}+B_{3 j} R_{3 j j}^{-1} B_{3 j}^{T} K_{j}\right\} .
$$


Then optimal trajectory $x^{*}(t)$ and the values of $u_{i}^{*}(t)$ and $u_{3 i}^{*}(t), i=1,2$, are given by

$$
\begin{aligned}
& x^{*}\left(t ; t_{0}, x_{0}\right)=\phi\left(t, t_{0}\right) x_{0}, \\
& u_{i}^{*}(t)=-R_{i i}^{-1} B_{i}^{T} K_{i} \phi\left(t, t_{0}\right) x_{0}, \\
& u_{3 i}^{*}(t)=-R_{3 i}^{-1} B_{3 i}^{T} K_{i} \phi\left(t, t_{0}\right) x_{0}, \\
& \quad i=1,2 .
\end{aligned}
$$

If we require

$$
\begin{aligned}
& \eta_{10}(t)=-R_{11}^{-1} B_{1}^{T} K_{1}+\eta_{11} R_{311}^{-1} B_{31}^{T} K_{1} \\
& \eta_{20}(t)=-R_{22}^{-1} B_{2}^{T} K_{2}+\eta_{22} R_{322}^{-1} B_{32}^{T} K_{2}
\end{aligned}
$$

then the equations (15) and (16) hold, i. e., we have

$$
\begin{aligned}
u_{1}(t) & =\bar{\gamma}_{1}\left(x(t), u_{31}(t), t\right) \\
& =\eta_{10}(t) x(t)+\eta_{11}(t) u_{31}(t), \\
u_{2}(t) & =\bar{\gamma}_{2}\left(x(t), u_{32}(t), t\right) \\
& =\eta_{20}(t) x(t)+\eta_{22}(t) u_{32}(t)
\end{aligned}
$$

where $\eta_{i 0}(t)$ and $\eta_{i i}(t), i=1,2$, satisfy (25) and (26).

(II) The solution of Problem (B).

For given $\eta_{i 0}(t)$, and $\eta_{i i}(t), i=1,2$, which satisfy (25) and (26), Problem (B) will be as follows

$$
\min J_{3}\left(u_{1}, u_{2}, u_{31}, u_{32}\right)
$$

$u_{31}, u_{32}$

$$
\begin{aligned}
\text { s.t. } \quad \dot{x}(t) & =A(t) x(t)+\sum_{i=1}^{2}\left\{B_{i}(t) u_{i}(t)+B_{3 i}(t) u_{3 i}(t)\right\} \\
x\left(t_{0}\right) & =x_{0}, \quad t \in\left[t_{0}, t_{1}\right], \\
& u_{1}(t)=\bar{\gamma}_{1}\left(x(t), u_{31}(t), t\right), \\
u_{2}(t) & =\bar{\gamma}_{2}\left(x(t), u_{32}(t), t\right), \\
& u_{3 i}(t) \in \boldsymbol{R}^{m_{3 i}}, \quad i=1,2 .
\end{aligned}
$$

Since $J_{3}$ is strictly convex in $u_{31}(t)$ and $u_{32}(t)$, by making use of the Pontryagin minimum principle, the necessary and sufficient conditions for Problem (B) are given by (11), and equations

$$
\left(\partial H / \partial u_{31}\right)=\eta_{11}^{T}\left\{R_{31} u_{1}+B_{1}^{T} \tilde{\lambda}\right\}+R_{331} u_{31}+B_{31}^{T} \tilde{\lambda}=0,
$$

$$
\left(\partial H / \partial u_{32}\right)=\eta{ }_{22}^{T}\left\{R_{32} u_{2}+B_{2}^{T} \tilde{\lambda}\right\}+R_{332} u_{32}+B_{32}^{T} \tilde{\lambda}=0,
$$

and

$$
(d \tilde{\lambda}(t) / d t)=-Q_{3} x(t)-A^{T} \tilde{\lambda}-\sum_{i=1}^{2} \eta_{i 0}^{T}\left\{R_{3 i} u_{i}+B_{i}^{T} \tilde{\lambda}\right\},
$$

$$
\tilde{\lambda}\left(t_{1}\right)=\left.F_{3}\left(t_{1}\right) x(t)\right|_{t_{1}}, \quad t \in\left[t_{0}, t_{1}\right],
$$

where $\tilde{\lambda}(t) \in \boldsymbol{R}^{n}$ is an adjoint vector function, and $H$ is Hamiltonian as follows.

$$
\begin{aligned}
H= & \frac{1}{2} \cdot\left\{x^{T}(t) Q_{3}(t) x(t)\right. \\
& \left.+\sum_{j=1}^{2}\left[u_{j}^{T}(t) R_{3 j}(t) u_{j}(t)+u_{3 j}^{T}(t) R_{33 j}(t) u_{3 j}(t)\right]\right\}
\end{aligned}
$$

$$
+\tilde{\lambda}^{T}(t)\left\{A(t) x(t)+\sum_{j=1}^{2}\left[B_{j}(t) u_{j}(t)+B_{3 j}(t) u_{3 j}(t)\right]\right\}
$$

(III) The conditions for the incentive Stackelberg strategies of $\mathbf{P 1}$ and $\mathbf{P 2}$.

Now, we will ensure that $u_{31}^{*}(t), u_{32}^{*}(t)$ and $x^{*}(t)$ are the solutions of Problem (B). For this, we need, together with (25) and (26), that they hold

$$
\begin{aligned}
& \eta_{11}^{T}\left\{R_{31} u_{1}^{*}+B_{1}^{T} \tilde{\lambda}\right\}+R_{331} u_{31}^{*}+B_{31}^{T} \tilde{\lambda}=0, \\
& \eta_{22}^{T}\left\{R_{32} u_{2}^{*}+B_{2}^{T} \tilde{\lambda}\right\}+R_{332} u_{32}^{*}+B_{32}^{T} \tilde{\lambda}=0,
\end{aligned}
$$

for all $t \in\left[t_{0}, t_{1}\right]$, where $\tilde{\lambda}(t)$ is the solution of the following differential equation

$$
\begin{aligned}
& \qquad \frac{d \tilde{\lambda}(t)}{d t}=-Q_{3} x^{*}(t)-A^{T} \tilde{\lambda}-\sum_{i=1}^{2} \eta_{i_{0}}\left\{R_{3 i} u_{i}^{*}+B_{i}^{T} \tilde{\lambda}\right\}, \\
& \tilde{\lambda}\left(t_{1}\right)=\left.F_{3}\left(t_{1}\right) x^{*}(t)\right|_{t_{1}}, \quad t \in\left[t_{0}, t_{1}\right] \\
& \text { Let } \\
& \tilde{\lambda}(t)=P(t) x^{*}(t) .
\end{aligned}
$$

Substituting respectively for $\tilde{\lambda}(t), x^{*}(t), u_{i}^{*}(t)$ and $u_{3 i}^{*}(t), i=1,2$, from (35), (22), (23), and (24) in (34), we can obtain that $P(t)$ will be the solution of the following linear matrix differential equation

$$
\begin{aligned}
& \dot{P}(t)=-P \Lambda-A^{T} P-Q_{3}-\sum_{i=1}^{2} \eta_{i 0}^{T}\left\{B_{i .}^{T} P-R_{3 i} R_{i i}^{-1} B_{i}^{T} K_{i}\right\} \\
& P\left(t_{1}\right)=F_{3}\left(t_{1}\right), \quad t \in\left[t_{0}, t_{1}\right] .
\end{aligned}
$$

Similarly, substituting respectively for $\tilde{\lambda}(t)$, $x^{*}(t), u_{i}^{*}(t)$ and $u_{3 i}^{*}(t), i=1,2$, from (35), (22) (24) in (32) and (33), we can also have

$$
\begin{aligned}
& \eta_{11}^{T}\left\{B_{1}^{T} P-R_{31} R_{11}^{-1} B_{1}^{T} K_{1}\right\}=R_{331} R_{311}^{-1} B_{31}^{T} K_{1}-B_{31}^{T} P, \\
& \eta_{22}^{T}\left\{B_{2}^{T} P-R_{32} R_{22}^{-1} B_{2}^{T} K_{2}\right\}=R_{332} R_{322}^{-1} B_{32}^{T} K_{2}-B_{32}^{T} P
\end{aligned}
$$

where, of course, $P(t)$ is the solution of the equation (36).

For the purpose of simplicity, here we further discuss (36). By making use of (25), (26), (37) and (38), we can eliminate $\eta_{i 0}(t), i=1,2$, from (36), and obtain directly the expression of the solution of $P(t)$. Thus, defining

$$
\begin{aligned}
Z(t)= & \sum_{i=1}^{2} K_{i}^{T}\left\{B_{i} R_{i \bar{i}}^{-1} R_{3 i} R_{\bar{i}}^{-1} B_{i}^{T}\right. \\
& \left.+B_{3 i} R_{3 i}^{-1} R_{33 i} R_{3 i}^{-1} B_{3 i}^{T}\right\} K_{i},
\end{aligned}
$$

we can have

$$
\begin{aligned}
& \dot{P}(t)=-P \Lambda-\Lambda^{T} P-Q_{3}-Z, \\
& P\left(t_{1}\right)=F_{3}\left(t_{1}\right), \quad t \in\left[t_{0}, t_{1}\right],
\end{aligned}
$$

where $\Lambda(t)$ has been defined in (21). 
Note that $P(t)$ is symmetric since $Z(t)$ is. Then, the solution of (40) will be given by

$$
\begin{aligned}
P\left(t ; t_{1}, F_{3}\right)= & \phi^{T}\left(t_{1}, t\right) F_{3}\left(t_{1}\right) \phi\left(t_{1}, t\right) \\
& +\int_{t}^{t_{1}} \phi^{T}(\tau, t)\left\{Q_{3}(\tau)+Z(\tau)\right\} \phi(\tau, t) d \tau .
\end{aligned}
$$

Thus if $\eta_{i 0}(t)$ and $\eta_{i i}(t), i=1,2$, satisfy (25), (26), (37) and (38), where $K_{i}(t), i=1,2$, and $P(t)$ are given by (19) and (41), respectively, then the following form of the strategies

$$
\begin{aligned}
& \bar{\gamma}_{1}\left(x(t), u_{31}(t), t\right)=\eta_{10}(t) x(t)+\eta_{11}(t) u_{31}(t), \\
& \bar{\gamma}_{2}\left(x(t), u_{32}(t), t\right)=\eta_{20}(t) x(t)+\eta_{22}(t) u_{32}(t),
\end{aligned}
$$

are the two-level incentive Stackelberg strategies of the players $\mathbf{P 1}$ and $\mathbf{P 2}$ in this two-level incentive differential game.

Then we obtain the following theorem with the two-level incentive Stackelberg strategies $\bar{\gamma}_{1} \in \boldsymbol{\Gamma}_{1}$ and $\bar{\gamma}_{2} \in \boldsymbol{\Gamma}_{2}$.

Theorem 1. Let $\eta_{i 0}(t) \in \boldsymbol{R}^{m_{i} \times n}$ and $\eta_{i i}(t) \in \boldsymbol{R}^{m_{i} \times m_{3 i}}$ $i=1,2$, satisfy respectively (25), (26), (37) and (38) for all $t \in\left[t_{0}, t_{1}\right]$, where $K_{i}(t), i=1,2$, and $P(t)$ are given by (19) and (41), respectively. Then the strategies $\bar{\gamma}_{1} \in \boldsymbol{\Gamma}_{1}$ and $\bar{\gamma}_{2} \in \boldsymbol{\Gamma}_{2}$, where

$$
\begin{aligned}
& \bar{\gamma}_{1}\left(x(t), u_{31}(t), t\right)=\eta_{10}(t) x(t)+\eta_{11}(t) u_{31}(t), \\
& \bar{\gamma}_{2}\left(x(t), u_{32}(t), t\right)=\eta_{20}(t) x(t)+\eta_{22}(t) u_{32}(t),
\end{aligned}
$$

are the two-level incentive Stackelberg strategies in the two-level incentive differential games with two noncooperative leaders and one follower, characterized by the linears state dynamics and quadratic cost functionals described in Section 3.

The proof is obvious from what was stated prior to this theorem.

\section{Remark :}

i) The two-level incentive Stackelberg strategies $\bar{\gamma}_{1} \in \boldsymbol{\Gamma}_{1}$ and $\bar{\gamma}_{2} \in \Gamma_{2}$ are linear for $x(t), u_{31}(t)$ and $u_{32}(t)$ in Theorem 1 .

ii) According to the procedures by which Theorem 1 was derived, we can know that this theorem is only the sufficient conditions for $\bar{\gamma}_{1}$ and $\bar{\gamma}_{2}$ being the two-level incentive Stackelberg strategies in such a differential game problem.

iii) By the procedures stated above, we can obtain the following order by which $\bar{\gamma}_{1} \in \Gamma_{1}$ and $\bar{\gamma}_{2} \in \boldsymbol{\Gamma}_{2}$ can be directly determined. We first calculate $K_{i}(t), i=1,2$, by the coupled matrix Riccati differential equations (19); however, $P(t)$ can directly be obtained by the formula (41) of the solution of the differential equation (40); then calculate $\eta_{i i}(t), i=1,2$, by (37) and (38) which only involve $K_{i}(t), i=1,2$, and $P(t)$; finally calculate directly $\eta_{i 0}(t), i=1,2$, by (25) and (26). According to this order, $\bar{\gamma}_{1} \in \boldsymbol{\Gamma}_{1}$ and $\bar{\gamma}_{2} \in \boldsymbol{\Gamma}_{2}$ are easily determined.

\section{Conclusion}

This paper mainly considers a two-level hierarchical differential game with two noncooperative leaders and one follower, characterized by a kind of linear state dynamics and quadratic cost functionals, which has not been considered in detail by the authors mentioned in Refs. 1) 11). In the present paper, we first define a concept of the "team-optimal Nash equilibrium solution". Then for the linear quadratic two-level incentive differential games with two noncooperative leaders and one follower in which two leaders find their "team-optimal Nash equilibrium solution" by announcing the corresponding incentive Stackelberg strategies, we construct these two-level incentive Stackelberg strategies $\bar{\gamma}_{1} \in \Gamma_{1}$ and $\bar{\gamma}_{2} \in \Gamma_{2}$ which are new form, and also obtain the sufficient conditions which are satisfied by $\bar{\gamma}_{1}$ and $\bar{\gamma}_{2}$.

As stated in this paper, $\bar{\gamma}_{1} \in \Gamma_{1}$ and $\bar{\gamma}_{2} \in \Gamma_{2}$ are respectively linear for $x(t), u_{31}(t)$ and $u_{32}(t)$ in such strategies. By the order of the calculation in this paper, $\bar{\gamma}_{1}$ and $\bar{\gamma}_{2}$ are easily determined, and the calculation is straightforward and feasible.

By making use of the approach and concept given in this paper, we also may obtain some results for a two-level incentive differential game with noncooperative $N$-leader $(N>2)$ and noncooperative $M$-follower, characterized by linear state dynamics and quadratic cost functionals. Of course, the approach given in this paper may be generalized to such an incentive differential game with two cooperative leaders and one follower, which will be further our research.

\section{References}

1) Y.C. Ho, Peter B. Luh and G. J. Olsder: A ControlTheoretic View on Incentives, Automatica, 18-2, 167/179 (1982)

2) T. Basar and H. Selbuz: Closed-Loop Stackelberg Strategies with Applications in the Optimal Control 
of Multilevel Systems, IEEE Trans. Automatic Control, AC-24-2, 166/178 (1979)

3) B. Tolwinski: Closed-Loop Stackelberg Solution to Multi-Stage Linear Quadratic Game, Journal of Optimization Theory and Applications, 34-3, 485/ 501 (1981)

4) Y.P. Zheng and T. Basar: Existence and Derivations of Optimal Affine Incentive Schemes for Stackelberg Games with Partial Information: A Geometric Approach, International Journal of Control, 35-6, 997/1011 (1982)

5) Y.P.Zheng, T. Basar and J. B. Cruz Jr.: Stackelberg Strategies and Incentives in Multiperson Deterministic Decision Problems, IEEE Trans. Systems, Man, and Cybernetics, SMC-14-1, 10/24 (1984)

6) T. Basar and G. J. Olsder: Team-Optimal ClosedLoop Stackelberg Strategies in Hierarchical Control Problems, Automatica, 16-3, 409/414 (1980)

7) Peter B. Luh, S. C. Chang and T.S. Chang: Solu- tions and Properties of Multi-Stage Stackelberg Games, Automatica, 20-2, 251/256 (1984)

8) T.S. Chang and Peter B. Luh: Derivation of Necessary and Sufficient Conditions for Single-State Stackelberg Games via the Inducible Region Concept, IEEE Trans. Automatic Control, AC-29-1, 63/66 (1984)

9) Peter B. Luh, T.S. Chang and T. Ning: ThreeLevel Stackelberg Decision Problems, IEEE Trans. Automatic Control, AC-29-3, 280/282 (1984)

10) T. Basar: Equilibrium Strategies in Dynamic Games with Multilevel of Hierarchy, Automatica, 17-5, 749/754 (1981)

11) T. Ishida and E. Shimemura: Three-Level Incentive Strategies in Differential. Games, International Journal of Control, 38-6, 1135/1148 (1983)

12) T. Basar and G.J. Olsder: Dynamic Noncooperative Game Theory, Academic Press, New York (1982) 